\title{
Artefact
}

Techniques, histoire et sciences humaines

\section{Du dessin de fabrique à la formation des dessinateurs : des techniciens entre art et industrie (XVIII ${ }^{\mathrm{e}}$-XIX ${ }^{\mathrm{e}}$ siècles)}

\section{Audrey Millet}

\section{(2) OpenEdition}

Journals

Édition électronique

URL : https://journals.openedition.org/artefact/9020

DOI : $10.4000 /$ artefact.9020

ISSN : 2606-9245

Éditeur :

Association Artefact. Techniques histoire et sciences humaines, Presses universitaires du Midi

Édition imprimée

Date de publication : 11 septembre 2014

Pagination : 75-88

ISBN : 978-2-271-08150-6

ISSN : 2273-0753

Référence électronique

Audrey Millet, «Du dessin de fabrique à la formation des dessinateurs : des techniciens entre art et industrie (xvIII-xIx siècles) », Artefact [En ligne], 2 | 2014, mis en ligne le 12 mai 2021, consulté le 24 août 2021. URL : http://journals.openedition.org/artefact/9020 ; DOI : https://doi.org/10.4000/ artefact. 9020

\section{(2) $\odot \Theta \Theta$}

Artefact, Techniques, histoire et sciences humaines est mise à disposition selon les termes de la Licence Creative Commons Attribution - Pas d'Utilisation Commerciale - Pas de Modification 4.0 International. 


\section{Du dessin de fabrique à la formation des dessinateurs: des techniciens entre art et industrie (xvIII ${ }^{\mathrm{e}} \mathrm{xIX} \mathrm{x}^{\mathrm{e}}$ siècles)}

Audrey MILLET ${ }^{1}$

\section{Résumé}

S'exonérer des taxinomies sociales ordinaires à propos de l'activité de dessinateur suppose de se garder de trois écueils, respectivement historiographique, documentaire et ethnologique. Il faut d'abord s'éloigner des oppositions binaires reprises par l'historien trop pressé: art/industrie, artiste/artisan, art libéral/art mécanique, dessin d'art/dessin technique. De ce point de vue, la formation technique des dessinateurs, personnel essentiel dans la compétition entre les manufacturiers, contribue par son institutionnalisation à la codification du métier de dessinateur. Mobiliser des sources continûment oubliées dans les réserves des musées permet ensuite de renouveler avec profit les perspectives, à la rencontre complexe de l'art, de la technique et de l'économie - et de leurs propres redéfinitions dans le travail d'esquisse et de mise en carte. Le caractère changeant du goût et des objets contraint à un apprentissage dans lequel la répétition du geste est une chorégraphie du dessinateur à la fois routinière et créatrice. À l'atelier, en école ou en manufacture, la première industrialisation épuise autant le geste que l'institution.

Mots-clés : art et industrie, dessin, dessinateur, formation, métier, technique.

\section{Abstract}

To exonerate from ordinary social taxonomies in connection with the activity of draughtsman supposes to avoid three pitfalls, respectively historiographical, documentary and ethnological. It is necessary to move away binary oppositions taken again by the impatient historian: art/industry, artist/craftsman, liberal art/mechanical art, drawing of technical art/drawing. From this point of view, the technical training of the draughtsmen, 
essential staff in the competition between the manufacturers, contributed by his institutionalization to the coding of the trade of draughtsman. Mobilizing sources continuously forgotten in the reserves of the museums then makes it possible to renew with profit the prospects, with the complex meeting of art, technique and economy - and their own redefinitions in the work of draft and mise-en-carte. The changing characteristics of taste and of objects constrained to a training in which the repetition of his task was a choregraphy of the draughtsman at the same time routine and creative. In the workshop, in school or in manufacture, the first industrialization exhausted the gesture as much as the institution.

Keywords: art and industry, draughtsman, drawing, technic, trade, training.

À la mémoire de Paul Duperray (2013) ingénieur des arts et de l'industrie textile, navette d'argent à Tours

Les représentations véhiculées par les hommes de la fabrique ont servi à construire une mythologie du dessinateur, artiste et technicien accompli². En effet, ces auteurs, nostalgiques d'un xvIII $^{e}$ siècle considéré comme l'âge d'or de la Grande Fabrique, dressent un portrait flatteur d'un dessinateur capable d'inventions et d'innovations ${ }^{3}$. Il participe d'une construction identitaire largement «lyonnocentrique» qui ne révèle en rien la réalité du métier et fait l'impasse sur les autres praticiens ${ }^{4}$. Cette image polie est une exception, voire un mythe. L'analyse des dessins et des pratiques nous permet d'approcher à la fois les permanences et l'hétérogénéité du métier. L'expression "dessin de fabrique» cache des esquisses, mises en cartes et papiers huilés, dont le point commun est de participer à la décoration des objets du quotidien, luxueux ou banals. Le statut de ces papiers est indéfini car l'historien les a considérés comme des traces du monde de l'art, à l'exception notable de Serge Chassagne ${ }^{6}$, et l'historien de l'art a sous-estimé l'in- térêt de cet art dit mineur ${ }^{7}$. La faute, sans doute, à leur lecture difficile, puisque ce sont des papiers techniques, et à l'utilisation de classements simplistes.

Propriétés des manufactures, aujourd'hui pour la plupart fermées, les dessins ont été éparpillés entre les musées, les centres d'archives, les particuliers, ou encore brûlés. Plutôt que de différencier dessins d'ornement et de formes, alors que l'on sait bien qu'un dessin pour textile n'est en aucun cas destiné à une surface plane-comment penser la rencontre de la chaîne et de la trame durant la fabrication, dans le cas contraire? -, pas plus qu'un dessin pour céramique ne se limite aux courbes - que l'on songe aux ornements sculptés, aux bulles apparues à la cuisson -, nous voudrions lier dessins, formation des dessinateurs et pratiques à partir de l'analyse d'une base de données de 2468 dessinateurs et peintres de fabrique ${ }^{8}$ (Annexe 1). Les archives prud'homales (à Paris) conservent bien quelques dessins déposés dans le cadre de la propriété industrielle, mais c'est au sein des musées que nous trouvons 
suffisamment de matériaux pour enquêter. Le musée des Arts décoratifs (à Paris) garde une centaine de livres $\mathrm{d}^{\prime}$ esquisses et de mises en cartes. ${ }^{9}$. La consultation du fonds de la manufacture Davenière, à la Cité internationale de la dentelle et de la mode (à Calais), et de l'école de dessin de la ville a été bénéfique. Le musée des beaux-arts de Tours conserve une partie du fonds de l'école de dessin (1776-1826) ${ }^{10}$. Enfin, les musées de l'impression sur étoffes à Mulhouse et du papier peint à Rixheim, ainsi que la Cité de la céramique de Sèvres, possèdent des modèles en grand nombre ${ }^{11}$. Ces sources permettent de couvrir le long $\mathrm{xVIII}^{\mathrm{e}}$ siècle et $\mathrm{d}^{\prime}$ aborder nos questions à partir d'un temps long, tout en prenant en compte des secteurs diversifiés (soie, céramique, papier peint...).

Un unique dessin peut fournir de nombreuses indications: motifs, inspirations et copies, exigences du support, de la machine, du fabricant et de la clientèle. Les commentaires, notes, refaits et ratés sont d'un grand intérêt et montrent, au-delà de la diversité des supports et destinations de la décoration, l'unité des pratiques. À rebours du temps de la création, ces graphes gardent les traces de la formation du dessinateur. Que nous apprennent les codes et conventions graphiques sur la formation? De quelles manières les dessins témoignent-ils des pratiques?

Face aux imprécisions des définitions, nous examinons les trois étapes $\mathrm{du}$ dessin de fabrique: esquisse, mise au net et dessin technique. Une analyse sous l'angle de la formation en atelier, en école et en manufacture est nécessaire pour appréhender le savoir-faire du dessinateur au quotidien dans un dernier point.

\section{Une vue de l'objet et des pratiques: esquisse, mise au net et mise en carte}

Quelle que soit la destination de la décoration - céramique, orfèvrerie ou encore soierie - Thominet à Nottingham, Buteux à Sèvres, Couder à Paris, Lebert à Mulhouse, utilisent un matériel commun pour traduire leur idée sous la forme $d^{\prime}$ une esquisse ${ }^{12}$. Dès la fin du $\mathrm{xvIII}^{\mathrm{e}}$ siècle, la majorité d'entre eux travaille sur des feuilles Canson Mongolfier $^{13}$, des papiers vélins ${ }^{14}$ et huilés ${ }^{15}$. Ils en connaissent les avantages et les inconvénients et utilisent des techniques picturales identiques ${ }^{16}$. Ces traces témoignent de la maîtrise de techniques et de matériaux communs sans qu'une distinction ne puisse être faite entre une application à la dentelle ou à la tapisserie, comme le montrent les modèles de César Galais, dessinateur en soierie et dentelles durant le $\mathrm{xIX}^{\mathrm{e}}$ siècle $^{17}$. De plus, les dessins préparatoires indiquent un même répertoire décoratif de végétaux d'ornement, mais les paysages, les scènes d'histoire, les architectures et les figures sont courants. Il est assez mal aisé d'indiquer à quelle finalité d'objet l'esquisse est destinée. «Dessin préparatoire», «esquisse», «croquis», «projet» 
sont les termes les plus utilisés ${ }^{18}$. La terminologie signale une unité dans la manière de désigner, dans la conception des dessins et du niveau de culture artistique des dessinateurs. Cependant, derrière ces termes se cache une autre réalité: celle de la fabrique. Jean-Baptiste Meusnier $^{19}$ ou Galais reprennent les proportions exactes de l'étoffe. Est donc connue la largeur d'évolution du dessin, c'est-à-dire le rapport, qui est la largeur égale à celle du chemin, donnée par le montage du métier, ou les dimensions de l'assiette. Des notes indiquent le type d'étoffe envisagé: "gros de Tours; broché; liseré20 ${ }^{20}$. De fait, l'esquisse est déjà un dessin appliqué. Le geste de Fragonard intègre donc cet horizon technique dès le premier dessin et l'étape suivante s'avère plus pointue encore ${ }^{21}$.

La mise au net, aussi appelée dessin de modèle, est l'étape intermédiaire entre l'esquisse et la mise en carte: elle vise à multiplier et agencer le motif dans le but de le retranscrire sur une feuille quadrillée ou non ${ }^{22}$. Dans le cas de la soierie, la mise au net se caractérise par la multiplication du motif qui prépare l'ordonnancement général de l'étoffe. Quant au dessinateur en dentelle, il multiplie sept fois l'esquisse pour réaliser sa mise au net. Les esquisses en dentelles de Thominet, de Moosberger ou de Richard montrent que trois traits sont nécessaires: le trait de tulle, le trait de guimpe et le trait de brodeur ${ }^{23}$. Ce papier de propreté exige un geste précis et appliqué; plus proche de la machine, il est habité des codes de l'industrie.

La mise en carte est un dessin arrêté, c'est-à-dire qualifié par l'arrêtage ${ }^{24}$. L'esquisse pour soierie adoptée, un dessinateur - dit aussi metteur en carte - la figure sur un papier quadrillé. Le but est de fixer par des conventions le mouvement des fils se croisant avec la trame afin de reproduire le dessin sur le tissu. Cette traduction par un langage conventionnel induit une connaissance de la technique du tissage. Les motifs peuvent être formés de trois façons différentes: par des effets de chaîne, des effets de trame ou des armures différentes du tissu lui-même, c'est-à-dire du fond. La mise en carte est une définition du métier à tisser qui ne permet pas d'approximations - comme celle de Pringot, illisible pour les fabricants eux-mêmes ${ }^{25}$. Dans le cas de l'impression, l'intermédiaire entre l'esquisse et l'objet tissé ou papier est la planche gravée. La difficulté technique réside dans le respect des pleins et des vides et dans le nombre de couleurs: «l'intermédiaire planche» nécessite une grande précision dans le dessin et une coopération suivie entre le dessinateur, le graveur et l'imprimeur. Dernier aspect technique à ne pas négliger: le volume des objets. En effet, Leloy, avant de penser la décoration d'une coupe, la décortique dans ses moindres détails pour en saisir les spécificités: volume, profondeur et relief ${ }^{26}$. Ce papier est une traduction en deux dimensions d'un produit en trois dimensions.

Le dessin de fabrique est le reflet des caractéristiques esthétique, technique et économique des manufactures. Les définitions sont aussi complexes que l'opération par laquelle le dessin appliqué et codifié, porteur de savoirs épars, formalise les techniques. Les étapes fournissent également des indications sur les pratiques et les gestes des dessinateurs. Pour Howard Becker, la coopération des acteurs suppose qu'ils partagent des 
conventions, c'est-à-dire des conceptions communes du travail qui, sauf dysfonctionnement de la coopération, n'ont pas besoin d'être explicitées. Ces conventions sont construites par la pratique ${ }^{27}$. Cette dernière peut aussi être expliquée comme un "ordre négociée ${ }^{28}$ » puisque certains dessinateurs ne s'appliquent qu'à la mise en carte, tandis que d'autres n'en réalisent jamais, comme le précise François Renaud ${ }^{29}$. Saisissant à la fois l'homogénéité et l'hétérogénéité des dessins et des gestes, il est possible d'envisager l'enjeu de formation des dessinateurs, à la fois simple et capital: faciliter les choix techniques des «hommes de l'art» et leur rendre accessibles des codes et conventions jusqu'alors partagés par les seuls "gens du métier ${ }^{30}$ ». Ces spécificités interrogent sur les lieux et les manières de l'acquisition des savoirfaire. Où et comment le dessinateur est-il formé aux techniques?

\section{Trois lieux de formation distincts et complémentaires : école, atelier et manufacture}

Les matériaux et techniques employés ainsi que le répertoire décoratif nous indiquent que l'apprentissage se fait dans l'atelier du peintre. L'apprenti s'exerce à dessiner et à peindre sous les yeux du maître. Il copie les maîtres d'après gravures puis s'intéresse aux plâtres. Lorsque son niveau est suffisant, il réalise des morceaux de peinture. Comme le prouve leur production, la plupart des dessinateurs sont peintres de fleurs et d'ornements. On connait la réputation vers 1780 de l'atelier de Berjon, représentant de l'école lyonnaise, encensé par son élève Augustin Thierriat ${ }^{31}$. Quant à Redouté, il organise autour du jardin botanique du Muséum d'histoire naturelle, créé en 1793, un atelier essentiellement composé de femmes et spécialisé dans les fleurs ${ }^{32}$. L'importance de la chaîne de coopération oblige à envisager une formation chez le graveur. Comme l'a montré Serge Chassagne, durant plusieurs mois, les établissements de Montaut et Laborde ne comptent pas de dessinateur parmi leurs salariés, tandis qu'on note la présence de graveurs en permanence ${ }^{33}$. Dès lors, on comprend mieux le nombre important de graveurs composant des dessins destinés à la lithographie ${ }^{34}$. Martin Riester a reçu une formation de graveur et travaille pour des éditeurs de grammaire d'ornements et pour des fabricants de toile imprimée autour de Mulhouse ${ }^{35}$. Comme le montre notre base de données, $16 \%$ des dessinateurs de fabrique sont aussi graveurs: Pierre Langevin, actif de 1773 à 1803 à Nantes, est dessinateur, manufacturier et graveur ${ }^{36}$. Malheureusement, il est difficile d'approcher cette formation: seules les planches sont gardées car elles sont indispensables au processus de fabrication, tandis qu'un dessin ne sert plus lorsqu'il a été gravé.

Envisager la formation en école n'a rien de surprenant; on connait l'École des arts décoratifs de Paris, mais il s'agit surtout de prendre en considération les 
enseignements des écoles gratuites de dessin qui se multiplient au XVIII ${ }^{\mathrm{e}}$ siècle $^{37}$. Perçues comme les petites sœurs de l'Académie royale, les professeurs y dispensent un enseignement en apparence assez similaire à celui de l'académie parisienne. L'apprentissage d'après copie est privilégiée $^{38}$, comme le montre le fonds de l'école de dessin de Tours créée en 1776 par Antoine Rougeot ${ }^{39}$. Au premier abord, les enseignements sont destinés aux artistes, mais le gendre et successeur de Rougeot, Jean-Jacques Raverot, donne à Galais une partie des dessins de l'école au début du xix ${ }^{\mathrm{e}}$ siècle $^{40}$. Aucun relevé d'architecture, aucun dessin de figure, ni académie n'apparaît dans les quarante-quatre albums $\mathrm{du}$ fonds Galais: il s'agit exclusivement d'esquisses, de mises au net et de mises en carte pour soieries et dentelles. De plus, Galais attribue des dessins à Barault, dessinateur pour la fabrique de Tours au $\mathrm{xVIII}^{\mathrm{e}}$ siècle et professeur d'ornements et de fleurs ${ }^{41}$. L'école forme des techniciens. Frédéric Morvan Becker l'a parfaitement résumé: c'est le «trompe-l'œil des Beaux-Arts ${ }^{42} »$.

Pour autant, il ne faut pas négliger la formation intégrée aux manufactures. Le système est précoce puisque c'est dès le $\mathrm{xVII}^{\mathrm{e}}$ siècle que la manufacture des Gobelins se dote d'une école ${ }^{43}$. Pourquoi investir dans la formation des dessinateurs si elle peut avoir lieu ailleurs? La main-d'œuvre qualifiée étant chère et instable, une formation intégrée à la manufacture avantage les dirigeants car elle permet de rémunérer à moindre coût les apprentis et de fixer le personnel. La formation est adaptée aux besoins de la manufacture, sans dépendre d'un enseignement toujours entaché du soupçon d'académisme. À Sèvres, des dessins réalisés d'après des modèles en plâtre et datant du troisième quart du $\mathrm{xvIII}^{\mathrm{e}}$ siècle sont encore conservés ${ }^{44}$. De plus, la majorité des peintres embauchés intègrent la manufacture un an avant la signature de leur contrat. Les dossiers du personnel indiquent $q^{\prime}{ }^{\prime} i l$ s'agit bien d'une formation en même temps que d'une mise à l'essai, à l'issue de laquelle ils sont remerciés ou recrutés ${ }^{45}$. L'intérêt d'une formation technique en manufacture est évident. Il n'y a ni métier à tisser, ni four au sein de l'atelier ou de l'école, alors que la fréquentation de ces équipements autorise l'œil à s'exercer et l'apprenti à s'imprégner de la routine de la fabrication. On se fie plus à cette formation in situ. Le fonds du fabricant de tulle Davenière témoigne du dialogue entre fabricant et dessinateur: «la pointe ne doit pas monter», «il y a beaucoup trop de choses dans le bouquet», «bien, il faut s'arranger à faire avec cela avec cette importance de détails» ou «Vous faites beaucoup trop de mouvements. Il m'est aussi impossible de rendre cette esquisse que de prendre la lune avec les dents. ${ }^{46}{ }^{\prime}$ Ces traces sont le reflet du quotidien de la fabrique. $75 \%$ des 2700 esquisses consultées datant de 1860 à 1900 sont longuement annotées, en particulier durant les vingt premières années de l'entreprise. C'est sans commune mesure avec $\mathrm{d}^{\prime}$ autres fonds ${ }^{47}$ car la récente industrie $\mathrm{du}$ tulle mécanique ne bénéficie d'aucune structure de formation au dessin. Une école d'art ouvre bien ses portes en 1841 à Saint-Pierre, mais elle ne dispense des leçons pour esquisses qu'en $1882^{48}$. Pour combler cette lacune, il n'est pas surprenant que Davenière forme des dessinateurs au sein de sa manufacture. 
La formation intégrée explique toutes ces annotations.

Atelier, école et manufacture, peinture et gravure sont les voies d'intégration des cabinets de dessin. La répétition $\mathrm{du}$ geste, la pratique, la copie se situent au centre de la formation permettant la compréhension opératoire de l'action des dessinateurs ${ }^{49}$. L'apprentissage du dessin est loin de se limiter aux structures traditionnelles corporatives. Lieux et manières sont complémentaires et montrent que le métier de dessinateur s'envisage comme le point de rencontre de l'art et de l'industrie, sans que l'un ne prenne l'ascendant sur l'autre. Les objets du long XviII ${ }^{\mathrm{e}}$ siècle ne sont pas dénués de recherches esthétiques et de talents, au contraire. Les dessins démontrent que l'art sert l'industrie et réciproquement, formant alors un régime de fabrique. Enfin, l'acquisition d'un savoir-faire durant l'exercice du métier doit être abordée.

\section{L'exercice du métier : un apprentissage au quotidien}

Derrière l'expression générique «dessinateur de fabrique» se cachent des trajectoires, des manières de travailler et des formations qui peuvent s'avérer très différentes. Comment composer, proposer et innover dans une activité supposée répétitive et routinière? Un élément a particulièrement retenu notre attention: les tampons de dessinateurs installés sous l'enseigne "cabinets de dessins». Leur accroissement est particulièrement remarquable dans les années 1830-1840. Les fabricants ne se contentent pas de la production de leur atelier de dessin et achètent régulièrement des modèles à l'extérieur. Pour alimenter leur industrie, les Calaisiens se fournissent à Paris ou à Nottingham ${ }^{50}$. Lorsqu'un dessinateur travaille en cabinet, il fournit un service au fabricant à qui il présente des dessins censés correspondre à l'esprit de la Maison. Son travail doit être varié, dans le style ou dans l'objet abordé (papier peint, châle, impression...). Ces structures méconnues ne peuvent-elles pas former des jeunes gens afin de les employer? Chez Prelle, à Lyon, le dessin n 4377 de 1852 a été réalisé «par Nelson chez Couderc fils ${ }^{51} »$. Ce dernier exerce le même métier que son père, Amédée: dessinateur pour fabrique. Nelson est salarié. «Cet établissement est, à vrai dire, une manufacture de dessins qui alimente» les fabricants en tous genres dès les années 1840 , tandis que d'autres sources mentionnent des ateliers de dessins individuels et collectifs dès $1820^{52}$. Toutefois, Malaine était déjà formateur au sein de son propre atelier à la fin du XvIII $^{e}$ siècle $^{53}$. S'il est probable que des dessinateurs soient formés «sur le tas» au sein d'ateliers privés, il semble important de s'interroger sur les modalités de cet apprentissage: quels types de sources ont-ils à leur disposition?

Galais collectionne des dessins de diverses origines car l'activité s'appuie en grande partie sur une connaissance des images qui stimule son travail et lui permet un nombreimportant de variations sur le même thème. Le fonds du fabricant Davenière confirme l'importance 
de l'accès à une bibliothèque graphique variée $^{54}$. Comme la bibliothèque de Philippe Oberkampf, à Jouy-en-Josas, ces sources d'inspiration sont installées dans le cabinet de dessins de la manufacture $^{55}$. Le dessinateur dispose de sources anciennes et des dernières tendances pour former son œil chaque jour. Cette formation cumulative concerne aussi les techniques $^{56}$. La participation du dessinateur de fabrique aux nouveaux procédés $\mathrm{d}$ 'impression montre qu'il a été formé durant son activité salariée. Entre 1835 et 1844, Fleury Chavant, dessinateur, inventeur de papier de mise en carte et éditeur, publie un nombre important d'ouvrages destinés à la fabrique. Le musée $d u$ dessinateur de fabrique, Fleurs et ornements pour papier peint, étoffes imprimées par des artistes spéciaux sont des ouvrages réalisés par Clerget, Lenfant et Buffardin ${ }^{57}$. Ces publications captent l'air du temps et montrent les capacités d'adaptation des dessinateurs. Dans un dernier point, des profils très différents peuvent remettre en cause l'appellation générique «dessinateur de fabrique».

Le cas de la création du service de Sèvres pour la laiterie de Rambouillet est révélateur. Le projet débute en 1785 et le comte d'Angiviller, directeur des bâtiments du roi, également responsable de la manufacture, met au point l'esprit du service de porcelaine. Louis Simon Boizot, chef de l'atelier de sculpture, dessine les formes, tandis que le peintre Jean-Jacques Lagrenée le Jeune, nommé directeur artistique associé, se charge de la décoration. Lagrenée doit «réformer le goût» et concevoir les décors, dont les dessins, rehaussés de gouache, sont encore aujourd'hui conservés dans les archives de la Cité de la céramique. Le nom de
Lagrenée cache en fait la multitude de dessinateurs et de peintres intervenus dans la réalisation du service. De nombreux dessins lui ont d'ailleurs été attribués à tort. Les conditions d'embauche de la manufacture - proximité géographique, membres des mêmes familles afin de fixer la main-d'œuvre - montrent que les peintres ont bénéficié d'une formation "sur le tas", très spécialisée, qui ne les autorise pas à prétendre à une position plus élevée au sein de la fabrique ou ailleurs. Les travaux sont d'un style médiocre mais la technique assez bien maîtrisée, ce qui semble pour l'instant suffire aux dirigeants. Une hiérarchie de l'activité de dessinateur, dont je ne propose pas une typologie définitive, est perceptible. Il se dégage provisoirement quatre positions: la direction artistique - à plusieurs têtes -, les chefs d'atelier, les peintres et le personnel de renfort. La formation seule n'explique pas cette répartition $^{58}$ : certains manufacturiers n'ont pas intérêt à mettre en place un système d'apprentissage qui leur coûterait du temps et de l'argent, permettant à la main d'œuvre de réclamer des appointements plus importants ou de les quitter. Il est bon d'avoir une armée de copistes et d'exécutants, compétents - mais pas trop ${ }^{59}$.

Aucune structure ne remplit toutes les conditions de formation, mais les leçons de dessin, les écoles gratuites, l'apprentissage en atelier et en manufacture indiquent les possibilités qui sont offertes aux aspirants dessinateurs. Néanmoins, c'est surtout notre regard qu'il s'agit de modifier. L'utilisation des termes art/ industrie, artiste/artisan, art libéral/ art industriel, dessert les historiens et 
sépare, de fait, deux mondes comme s'il s'agissait de sphères imperméables, sans jamais trouver un point de rencontre, sans qu'elles n'emploient les mêmes signes et sans que les travailleurs ne se fréquentent. Celina Fox a bien démontré qu'une séparation en deux sphères a été tentée à partir $d u x{ }^{e} I^{e}$ siècle, mais que celle-ci n'a pas duré6 ${ }^{60}$. Il s'agit plutôt de parler de «désunion» durant les années 1650-1750, puis d'une ré-union durant les $\mathrm{XVIII}^{\mathrm{e}}$ et $\mathrm{XIX}^{\mathrm{e}}$ siècles. Toutefois, cette désunion est-elle vraiment ressentie par la foule des exécutants? Ou n'est-ce que le trompe-l'œil des discours les plus visibles? L'analyse des dessins et des parcours de dessinateurs sous l'angle de la formation et de la technique permet de dépasser ces oppositions hâtives. Selon la position des dessinateurs, il n'est pas obligatoire de savoir réaliser une esquisse, une mise au net et une mise en carte, ni de connaître le montage d'un métier. Si la profession de metteur en carte, hautement technique, existe, c'est bien parce que tous les dessinateurs ne réalisent pas ce papier. Le peintre anonyme de la foule d'exécutants de Sèvres occupe, quant à lui, une place qu'il gardera toute sa vie. La formation dépend aussi des besoins des entreprises selon le contexte artistique et économique. Durant le long
$\mathrm{XVIII}^{\mathrm{e}}$ siècle, l'apprentissage montre que la répétition du geste et la chorégraphie des mains installent le dessinateur dans une routine. Celle-ci se qualifie par des actions (s'asseoir à son pupitre...), des savoir-faire informels et tacites, la mâ̂trise d'un matériel et la possession d'un bagage d'images mentales de l'exécutant, ce que Lucien Febvre appelle «l'outillage mental ${ }^{61} »$. Combines, décalques, coups de gomme, bricolages, arrangements de raccords, et compréhension d'une situation de création associent en permanence routine et invention. Quotidiennement, il peut passer de la gravure à l'impression, du tissage à la peinture: non pas que sa formation lui suffise pour aborder tous les supports, mais ses bases techniques lui permettent de questionner son propre savoir-faire et de l'adapter. Sa chôré est identique chaque jour, en même temps qu'elle se modifie, telle une routine formatrice qui permet de refuser toute vision mécaniste de la formation et des profils. L'art est technique en régime de fabrique et la technique se définit par une chaîne de coopération complexe qui engage aujourd'hui le chercheur à saisir la compréhension opératoire de l'action des dessinateurs par la mise en place d'un langage global du dessin, une esthétique scientifique. 


\title{
Annexe I: Dessinateurs en indiennes (1738-1773) Échantillon simplifié de la base de données
}

\author{
Légende \\ d. = dessinateur \\ p.d. = peintre dessinateur \\ g. = graveur \\ Manuf. = manufacturier
}

\section{Sources consultées}

Je remercie Monsieur Serge Chassagne, professeur émérite d'histoire à l'université de Lyon 2, pour la consultation de ses recherches sous forme de fiches, résultats de nombreuses investigations dans les archives notariales.

\begin{tabular}{|c|c|}
\hline $\begin{array}{l}\text { Archives de Paris } \\
\text { Série V.E: Registres paroissiaux et état civil } \\
\text { reconstitués (microfilmés et numérisés) } \\
\text { sous-séries 5Mi1, 5Mi2, 5Mi5 et V.5E, V.5Ebis }\end{array}$ & $\begin{array}{l}\text { Archives départementales de l'Oise, Beauvais } \\
2 \text { MITD/A1, tables décennales, 1824-1902 } \\
\text { (microfilms) } \\
\text { Série E-Registres paroissiaux et d'état civil, } \\
\text { tables décennales antérieurs à 1903 } \\
\text { sous-série 3 E: } 1 / 2 \text { à } 1 / 7 \text { (1752-1863) }\end{array}$ \\
\hline $\begin{array}{l}\text { Archives départementales de la Seine- } \\
\text { Maritime, Rouen } \\
\text { Série E-Notaires, communes, état civil, officiers } \\
\text { publics et ministériels (série E) } \\
\text { sous-série } 3 \text { E: archives communales } \\
\text { déposées } \\
\text { sous-série } 4 \text { E: état civil }\end{array}$ & $\begin{array}{l}\text { Archives départementales du Maine-et-Loire, } \\
\text { Angers } \\
\text { Série E-Fonds notariaux et état civil } \\
\text { sous-série 5 E: notaires } \\
\text { sous-série 6 E: registres paroissiaux et d'état } \\
\text { civil }\end{array}$ \\
\hline $\begin{array}{l}\text { Archives départementales du Haut-Rhin, } \\
\text { Mulhouse } \\
\text { Série E-Fonds notariaux et état civil } \\
\text { sous-série } 1 \text { E: état civil } \\
\text { sous-série 2 E: notariat }\end{array}$ & $\begin{array}{l}\text { Archives départementales de la Loire- } \\
\text { Atlantique, Nantes } \\
\begin{array}{l}\text { Série E-Fonds notariaux et état civil } \\
\text { sous-série } 3 \text { E: registres paroissiaux et l'état } \\
\text { civil }\end{array} \\
\end{array}$ \\
\hline $\begin{array}{l}\text { Archives départementales du Rhône, Lyon } \\
\text { Série E, Fonds notariaux et état civil } \\
\text { sous-série } 3 \mathrm{E} \text { : actes notariés (contrats de } \\
\text { mariages, testaments...) }\end{array}$ & $\begin{array}{l}\text { Archives départementales des Yvelines, } \\
\text { Montigny-le-Bretonneux } \\
\text { Série E-Fonds notariaux et état civil } \\
\text { sous-série 4 E: baptêmes et naissances, } \\
\text { mariages, sépultures et décès, tables } \\
\text { décennales }\end{array}$ \\
\hline $\begin{array}{l}\text { Archives départementales du Vaucluse, } \\
\text { Orange } \\
\text { Série E, Fonds notariaux et état civil } \\
\text { sous-série } 1 \mathrm{E} \text { : registres paroissiaux et d'état } \\
\text { civil } \\
\text { sous-série 3 E: notaires }\end{array}$ & \\
\hline
\end{tabular}




\begin{tabular}{|c|c|c|c|c|c|c|c|c|c|c|c|c|c|c|c|c|c|c|c|c|c|}
\hline 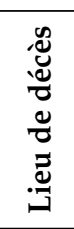 & 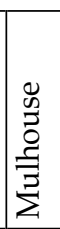 & & & & & & & 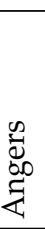 & & & & & & & & & & 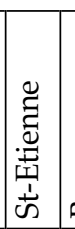 & 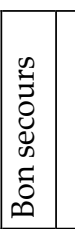 & & \\
\hline 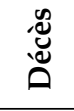 & 苋 & & & 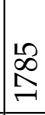 & & & & $\overrightarrow{\vec{\sigma}}$ & & & & & & & & & & 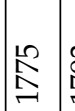 & શ્ & & \\
\hline 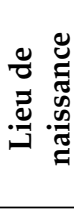 & 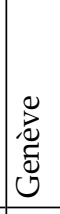 & & \begin{tabular}{|c}
5 \\
0 \\
$\vdots$ \\
\end{tabular} & 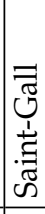 & & 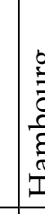 & & $\stackrel{\infty}{\Xi}$ & & 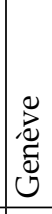 & & 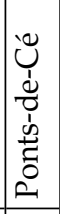 & 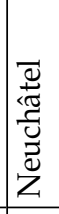 & 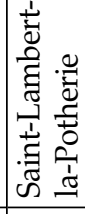 & 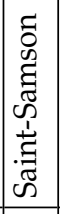 & 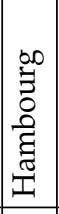 & & & 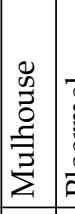 & 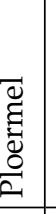 & \\
\hline $\begin{array}{l}\tilde{\Xi} \\
\ddot{z}\end{array}$ & 코 & & $\underset{\mathbb{N}}{\mathbb{N}}$ & 늘 & $\frac{a}{\infty}$ & & & ָָ & & & & & & 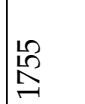 & & & 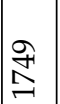 & & $\stackrel{\text { L }}{\stackrel{9}{\mathrm{~A}}}$ & & న̂ \\
\hline$\underset{\stackrel{N}{*}}{\stackrel{N}{*}}$ & & & 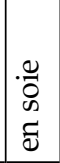 & & & & & & & & & & & & & & 0.0 & $\begin{array}{l}\overrightarrow{0} \\
\dot{2}\end{array}$ & & 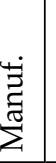 & \\
\hline 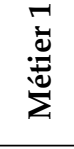 & ठ் & - & ர் & ரे & 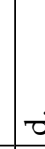 & $\tau$ & & ర் & & ن & os & ర் & ர் & ن & ن & ن & $\dot{2}_{1}$ & - & - & ர் & نं \\
\hline$\stackrel{\stackrel{0}{5}}{5}$ & 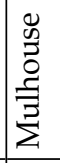 & 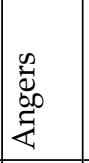 & 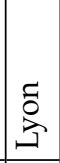 & 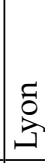 & 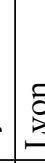 & $\begin{array}{l}\delta \\
\frac{g}{\partial} \\
\frac{\partial}{z} \\
z\end{array}$ & & 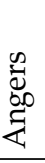 & 를 & 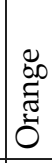 & 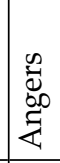 & 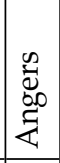 & 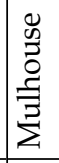 & 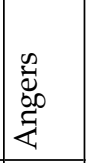 & 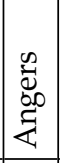 & 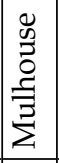 & 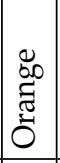 & 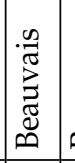 & 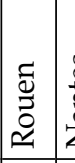 & 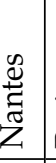 & 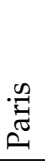 \\
\hline 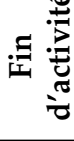 & 苋 & & & & & $\begin{array}{c}\infty \\
1 \\
\end{array}$ & & $\begin{array}{l}\infty \\
\stackrel{1}{1} \\
\end{array}$ & $\stackrel{8}{8}$ & $\stackrel{\infty}{\infty}$ & & $\begin{array}{l}\infty \\
\stackrel{D}{-} \\
-1\end{array}$ & & & 尽 & $\begin{array}{l}\infty \\
\stackrel{D}{-1}\end{array}$ & & 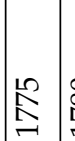 & 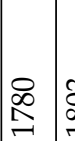 & $\begin{array}{l}8 \\
\infty \\
\infty \\
-1\end{array}$ & \\
\hline 苛 & 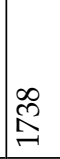 & 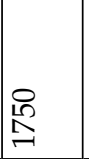 & 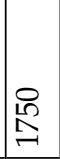 & 点 & กิ & 남 & & ڤ્ & $\stackrel{8}{8}$ & $\stackrel{8}{8}$ & 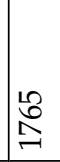 & 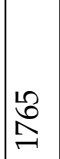 & $\stackrel{8}{2}$ & \begin{tabular}{l}
8 \\
\multirow{2}{2}{}
\end{tabular} & $\stackrel{ }{\stackrel{0}{2}}$ & 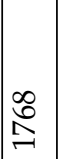 & 尽 & 尽 & 点 & $\stackrel{\Omega}{\Sigma}$ & 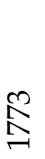 \\
\hline 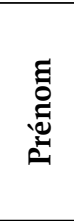 & $\stackrel{f i}{\rightleftarrows}$ & 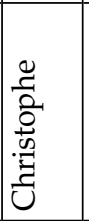 & 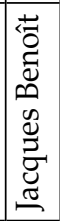 & 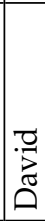 & 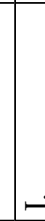 & 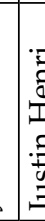 & & : & 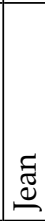 & 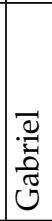 & 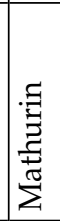 & 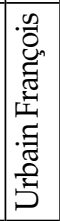 & 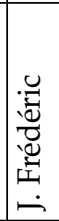 & 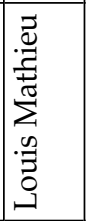 & 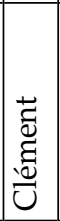 & 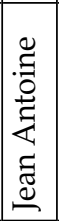 & 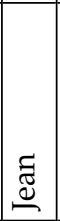 & 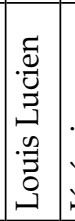 & : & 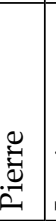 & .气 \\
\hline z̊̆ & 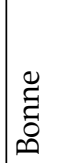 & 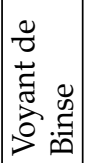 & & 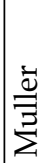 & 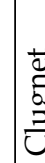 & $\frac{\pi}{2}$ & & 空 & 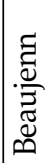 & $\begin{array}{l}\frac{n}{8} \\
\frac{0}{7} \\
\tilde{0}\end{array}$ & 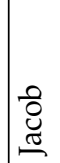 & 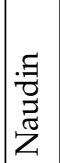 & 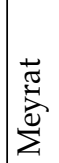 & 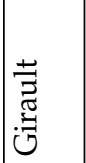 & 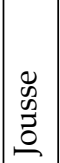 & $\mid \begin{array}{l}\frac{\pi}{0} \\
0 \\
\underline{j}\end{array}$ & $\vec{\pi}$ & 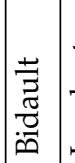 & 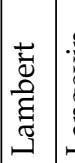 & 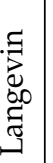 & . \\
\hline
\end{tabular}




\section{Notes}

1. Audrey Millet est doctorante contractuelle en histoire moderne sous la direction de Philippe Minard et d'Olivier Christin en cotutelle entre les universités de Paris 8 Vincennes-Saint-Denis et de Neuchâtel. Elle travaille sur les dessinateurs de manufactures durant le long $\mathrm{xvIII}^{\mathrm{e}}$ siècle. Elle est l'auteure d'articles sur la formation des dessinateurs, le genre de l'ornement à la manufacture de Sèvres et l'imitation dans la conception des dessins de mode. Contact: audreypatrizia@yahoo.fr.

2. Nous pensons à Joubert de l'Hiberderie, Guichard, Natalis Rondot et Émile Leroudier (É. Leroudier, Les cahiers d'Étienne Benoit (17611771). Livre de raison d'un dessinateur de la Fabrique lyonnaise, Lyon, M. Camus et Carnet, 1930).

3. Florence Charpigny a étudié le dessinateur de fabrique idéal de Leroudier, «Lyon et la Fabrique de soieries vus du $\mathrm{xx}^{\mathrm{e}}$ siècle: le journal apocryphe d'un dessinateur en soie», dans MariaAnne Privat-Savigny (dir.), Lyon au XVIII', un siècle surprenant!, Paris, Somogy/Lyon, Gadagne musées, 2012, p. 127-133; et «Les dessinateurs en soieries et la Fabrique Lyonnaise au XIX ${ }^{\mathrm{e}}$ siècle: histoire, représentations », Bulletin du CIETA, 1993, $\mathrm{n}^{\circ}$ 71, p. 121-137.

4. Pierre-Yves Saunier, L'esprit lyonnais $\mathrm{XIX}^{e}-\mathrm{XX}$ siècle. Genèse d'une représentation sociale, Paris, CNRS Éditions, 1995.

5. Les professions n'ont pas l'homogénéité que les sociologues fonctionnalistes ont bien voulu leur prêter: leur vision ne prend pas en compte l'irréductible diversité du réel. Parsons, Bucher et Strauss, à dix ans d'écart, insistent sur la nécessité d'étudier l'unité et la diversité des groupes professionnels et des pratiques de leurs membres. Talcott Parsons, The social systems, Glencoe, Free Press Year, 1951; Rue Bucher, Anselm Strauss, "Professions in process", American Journal of Sociology, vol. 66, no 4, janvier 1961, p. 325-334.

6. Serge Chassagne, La manufacture de toiles imprimées de Tournemine-lès-Angers (1752-1820) : étude d'une entreprise et d'une industrie au XVIII' siècle, Paris, C. Klincksieck, 1971.

7. Au sujet du mineur et du majeur, Gilles Deleuze, «Philosophie et minorité», Critique, n 34,1978, p. 155.

8. Le corpus des dessinateurs est aussi important que celui des dessins. Ce travail a nécessité une base de données réunissant aujourd'hui 634 dessinateurs en indienne, 315 dessinateurs en soierie, 172 en papier peint, 398 pour la céramique, 45 dessinateurs en dentelle, et 904 dessinateurs, personnel de renfort.
9. La bibliothèque des Arts décoratifs garde également le fonds Maciet. Jules Maciet (18461911), plus philanthrope que collectionneur, fait don de ses œuvres pour l'éducation artistique de tous. Hanté par la propagation de l'art, il accomplit un travail documentaire considérable, découpant des centaines de volumes pour en extraire les images et les classer par séries méthodiques.

10. L'incendie de 1940 a détruit une partie du fonds.

11. Le caractère composite du corpus est dû au statut complexe de ces papiers, à la fois archives et dessins d'art.

12. Au XVIII $^{\mathrm{e}}$ siècle, à Sèvres, les mêmes peintres et dessinateurs travaillent aux ornements, aux formes et aux finitions, tout comme Amédée Jean-Baptiste Couder (1797-1864) à Paris, responsable d'une manufacture de dessins: "Chaque jour, de nombreuses créations pour l'orfèvrerie, les bronzes, l'ébénisterie, les tapis, papiers peints, châles, soieries, robes peintes, etc. » sortent de son entreprise. En effet, on retrouve ses productions aussi bien dans le secteur de la soierie, chez Prelle, que dans la tapisserie, à Aubusson (Rapport du Jury central sur les produits de l'industrie française, Paris, Faim et Thunot, 1844, p. 389).

13. Leonard N. Rosenband, Papermaking in Eighteenth-Century France. Management, Labor and Revolution at the Montgolfier Mill, 1761-1805, Baltimore, The John Hopkins University Press, 2000.

14. Les Didot, imprimeurs de l'Institut de France, Bibliothèque de l'Institut, 12 septembre-15 décembre 2005, catalogue d'exposition, www.institut-de-france.fr.

15. Musée des Arts décoratifs, Paris (MAD), fonds Galais, vol. 41, p. 13.

16. Détail projet de toile imprimée, 1785 , manufacture de Jouy, plume encre brune, lavis gris et brun (MAD); esquisse pour soierie, $\mathrm{xVIII}^{\mathrm{e}}$ siècle, gouache sur papier, Musée des beaux-arts, Tours (MBA); esquisse dentelle, par Henry Ball, fin $\mathrm{XIX}^{\mathrm{e}}$ siècle, Cité internationale de la dentelle et de la mode, Calais (CIDM); étude pour le service de Louis-Philippe, Baptême de Louis XIII, Évariste Fragonard, gouache, début du XIX siècle, Cité de la céramique, Sèvres (CC); projet de papier peint, $1^{\text {re }}$ moitié du XIX ${ }^{\mathrm{e}}$ siècle, lavis d'encre de Chine et d'aquarelle (MAD).

17. Esquisses, fonds Galais (MAD), 44 vol.

18. Analyse réalisée à partir des esquisses destinées à la céramique de 1740 à 1880 (CC), des livres de croquis de la maison Prelle (xIx ${ }^{\mathrm{e}}$ siècle), des dessins et modèles du fonds Davenière (CIDM) et des mises en carte et esquisses du fonds Galais (MAD).

19. AD Rhône, 8B1043, dossier Meusnier, 
esquisses; et contrat de travail de Meusnier, dessinateur chez Aymard et Teste, 21 janvier 1761.

20. Esquisse, fonds Galais (MAD), vol. 41.

21. Ob 5, dossier Fragonard Jean Alexandre Évariste, dessinateur, peintre, sculpteur, travaux, 1806-1842 (CC).

22. Mise au net, fonds Galais (MAD), vol. 41, p. 17-18.

23. CIDM, fonds Davenière. Je remercie les tullistes de la CIDM de Calais pour leurs précieuses explications.

24. Claude Villard, Manuel de théorie du tissage,

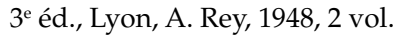

25. AD Indre-et-Loire, 9M17, Tours, 31 juillet 1816 .

26. La coupe est commandée en juillet 1840, Leloy (CC).

27. Howard Becker, Les mondes de l'art, Paris, Flammarion, 1988; voir la contribution de Pierre-Michel Menger dans la première édition, Art Worlds, Berkeley, 1982, p. 5.

28. George Herbert Mead, Movements of thought in the Nineteenth Century, M. H. Moore, University of Chicago Press, 1936, p. 360-361, cité par Strauss Anselm, La trame de la négociation, Paris, L'Harmattan, 1992, p. 88.

29. AM Lyon, 1C288, procédure Farcot, interrogatoire Renaud, 14 août 1737.

30. Pascal Dubourg-Glatigny et Hélène Vérin (dir.), Réduire en art. La technologie de la Renaissance aux Lumières, Paris, Maison des sciences de l'homme, 2008.

31. Berjon possède les connaissances suffisantes pour enseigner le dessin technique aux jeunes gens qui souhaiteraient s'orienter vers la Grande Fabrique.

32. Il emploie des élèves pour dessiner dans ses ouvrages et nous les retrouvons comme peintres à Sèvres, Pierre-Joseph Redouté, Les Liliacées, Paris, Didot Jeune, 1802-1816.

33. Serge Chassagne, «Du nouveau sur un atelier de toiles peintes à Angers (1763-1807), contemporain de la manufacture de Tournemine", Annales de Bretagne et des pays de l'Ouest, t. 83, $\mathrm{n}^{\circ} 1$, 1976, p. 167-185.

34. L'utilisation de la lithographie participe à la diffusion des modèles. Comparativement aux techniques de gravure que l'on n'acquiert qu'après un long apprentissage, son succès tient à sa facilité d'exécution. Florence Ott, «L'action de la Société industrielle de Mulhouse en faveur de l'enseignement professionnel», dans Brigitte Carrier-Reynaud (dir.), L'enseignement professionnel et la formation technique du début du XIX ${ }^{e}$ au milieu $d u$ $\mathrm{XX}^{e}$ siècle, Saint-Étienne, Publications de l'Université de Saint-Étienne, 2006, p. 24-31.
35. Véronique de La Hougue, Art et artistes du papier-peint en France: répertoire alphabétique, Montreuil, Gourcuff Gradenigo, 2007.

36. À son mariage, le 15 avril 1771, il est dit graveur et manufacturier (AM Nantes, registre GG755); en 1773, le dessinateur Merveillac travaille pour Langevin; entre 1799 et 1803, il travaille comme dessinateur au faubourg Saint-Jacques, quartier de la manufacture de Tournemine. Il est aussi graveur. S. Chassagne, La manufacture de toiles imprimées de Tournemine-lès-Angers..., op . cit., p. 276 et 370 .

37. Agnès Philouze-Lahalle, Les écoles de dessin au XVIII siècle : entre arts libéraux et arts mécaniques, Rennes, Presses universitaires de Rennes, 2006.

38. Académie, prix de satisfaction à Moreau à la distribution faite à l'École Centrale, 19 fructidor l'an IV, Rougeot, inv. 1939-802-2, G. Demarteau, d'après Van Loo; Étude de tête de femme, gravure à la manière d'une sanguine, "classe de figure", avril 1784, Rougeot, inv. 1939-802-1, Lingée, d'après Greuze.

39. Annie Gilet (dir.), Dessins $X V^{e}-X X^{e}$ siècle. La collection du musée de Tours, Tours, Musée des beaux-arts de Tours, 2002.

40. Cette partie n'est pas comprise dans le relevé d'Anatole Montaiglon réalisé postérieurement. A. Millet, «Charles-Antoine Rougeot et Jean-Jacques Raverot: itinéraire d'une famille au sein des écoles de dessin de Tours (1776-1826)», dans Virginie Fonteneau et Renaud d'Enfert (dir.), Espaces de l'enseignement scientifique et technique. Acteurs, savoirs, institutions, XVII ${ }^{e}-X^{e}$ siècles, Paris, Hermann, 2011, p. 109-118.

41. BAD 4983 (CC 109.22), MAD, Paris.

42. Frédéric Morvan, L'école gratuite de dessin de Rouen ou la formation des techniciens au XVIII siècle, thèse de doctorat, histoire moderne, sous la dir. de Philippe Minard, Université Paris 8 VincennesSaint-Denis, 2010; Renaud d'Enfert, L'enseignement du dessin en France. Figure humaine et dessin géométrique (1750-1850), Paris, Belin, 2003; id., Un art pour tous. Le dessin à l'école de 1800 à nos jours, Lyon, INRP, 2004; Renaud d'Enfert, Rossella Froissart, Ulrich Leben et Sylvie Martin, Histoire de l'École nationale supérieure des arts décoratifs (17661941), Paris, ENSAD, 2004.

43. Fr. Morvan, L'école gratuite de dessin de Rouen..., op. cit., p. 152-159.

44. Dessin, Étude de pied, Duplessis (atelier), Sèvres, $3^{\mathrm{e}}$ quart du $\mathrm{XVIII}^{\mathrm{e}}$ siècle, d'après un modèle en plâtre, craie, pierre, marque estampée, au verso à l'encre, CC.

45. CC, série D, registres du personnel, D1, D2 et D3. 
46. Esquisses pour un même motif, annotées, vers 1890, mine graphite et crayon de couleur, CIDM, fonds Davenière.

47. Le fonds Davenière est comparé aux données des établissements Prelle (soie), Réveillon et Zuber (papier peint).

48. Laurent Buchard, Benoît Noël, L'école d'art de Calais: arts, industrie et politique $d u \mathrm{XVIII}^{e}$ siècle à nos jours, Calais, Communauté d'agglomération du Calaisis, 2007.

49. Le format de l'article ne permet de développer ce thème, on se réfère donc au travail de Liliane Hilaire-Pérez, La pièce et le geste. Artisans, marchands et savoir technique à Londres au $\mathrm{XVIII}^{e}$ siècle, Paris, Albin Michel, 2013. Il s'agirait aussi de discuter de la mise en place d'une propriété des dessins de fabrique qui, contre toute attente, débute par une ordonnance consulaire de 1711 à Lyon (BM Lyon, imprimé 113884). On assiste ensuite à une escalade législative, conséquence de la multiplication et de la disponibilité des «choses banales». A. Millet, «Les normes intellectuelles et artistiques comme écho à l'esprit du capitalisme ou l'impossible propriété des modèles de fabrique ? France-Angleterre (xvIII ${ }^{\mathrm{e}-}$ XIX ${ }^{\mathrm{e}}$ siècles)", à paraître.

50. CIDM, fonds Davenière.

51. Échantillon, 15/07/1852, archives Prelle, patrons de modèles, livre $3, n^{\circ} 4377$.

52. L'Artiste, 1839, p. 209.

53. Bernard Jacqué, De la manufacture au mur: pour une histoire matérielle du papier peint (17741914), thèse de doctorat, histoire moderne, ss. la dir. de Serge Chassagne, Université Louis Lumière (Lyon 2), 2003, vol. 1.

54. 947 registres d'échantillons de l'entreprise à Calais, à Paris et des productions de Nottingham, Plauen ou Saint-Gall (dentelles, broderies, soieries, tissus imprimés), soit 100000 échantillons, 12 volumes de photographies... Pour une description du fonds, "Dessiner en régime de fabrique: l'imitation au cœur du processus créatif», Konsthistorisk tidskrift/Journal of Art History, Fashion and the Print: Translation and Transformation, Routledge, Taylor \& Francis, 2013, vol. 82, Issue 3, p. 272-286.

55. Serge Chassagne, Oberkampf. Un entrepreneur capitaliste au Siècle des Lumières, Paris, Aubier Montaigne, 1980. La bibliothèque a été déplacée de sa propre chambre au cabinet de dessins. Les dossiers de faillite des Archives de Paris montrent aussi un nombre très important de dessins.

56. A. Millet, «Dessiner en régime de fabrique...», op. cit.

57. Les recueils sont conçus par des dessinateurs pour des dessinateurs. Si les dessinateurs se sont facilement intégrés au commerce d'art de Chavant, c'est parce que le contexte est propice au développement de ces ouvrages peu onéreux, qu'ils travaillent dans le même quartier, voire la même rue de Cléry, que l'éditeur et qu'ils ont joué le jeu d'un rapide apprentissage sur le tas.

58. Il est bien dangereux de proposer une hiérarchie des peintres et dessinateurs. Toutefois, l'analyse des formes et contextes de la composition des salaires entre 1750 et 1860 concorde même si ces catégories restent perméables.

59. Sur l'imitation dans le processus créatif, A. Millet, «Dessiner en régime de fabrique...», op. cit.

60. Celina Fox, The arts of industry in the age of Enlightenment, Yale, Yale UniversityPress, 2010.

61. Lucien Febvre, Le problème de l'incroyance au XVI siècle. La religion de Rabelais, Paris, Albin Michel, 1942. 